\title{
Expression of plasma IFN signaling-related miRNAs during acute SARS-CoV-2 infection and its association with RBD-IgG antibody response
}

Jing $\mathrm{Wu}^{1 \dagger}{ }^{\dagger}$, Xingxiang $\mathrm{Liu}^{2 \dagger}$, Jianguo Shao ${ }^{3 \dagger}$, Yuanyuan Zhang ${ }^{2}$, Renfei $\mathrm{Lu}^{3}$, Hong Xue ${ }^{3}$, Yunfang Xu ${ }^{2}$, Lijuan Wang ${ }^{2}$, Hui Zhou ${ }^{4}$, Lugang Yu ${ }^{4}$, Ming Yue ${ }^{5^{*}}$ and Chen Dong ${ }^{1 *}$

\begin{abstract}
Background: Coronavirus disease 2019 (COVID-19) is a huge challenge worldwide. Although previous studies have suggested that type I interferon (IFN-I) could inhibit the virus replication, the expression characteristics of IFN-I signaling-related miRNAs (ISR-miRNAs) during acute severe acute respiratory syndrome coronavirus-2 (SARS-CoV-2) infection and its relationship with receptor-binding domain (RBD) $\lg G$ antibody response at the recovery phase remain unclear.

Methods: Expression profiles of 12 plasma ISR-miRNAs in COVID-19 patients and healthy controls were analyzed using RT-qPCR. The level of RBD-IgG antibody was determined using the competitive ELISA. Spearman correlation was done to measure the associations of plasma ISR-miRNAs with clinical characteristics during acute SARS-CoV-2 infection and RBD-IgG antibody response at the recovery phase.

Results: Compared with the healthy controls, COVID-19 patients exhibited higher levels of miR-29b-3p $(Z=3.15$, $P=0.002)$ and miR-1246 ( $Z=4.98, P<0.001)$. However, the expression of miR-186-5p and miR-15a-5p were significantly decreased. As the results shown, miR-30b-5p was negatively correlated with CD4+T cell counts $(r=-0.41$, $P=0.027)$ and marginally positively correlated with fasting plasma glucose in COVID-19 patients $(r=0.37, P=0.052)$. The competitive ELISA analysis showed the plasma level of miR-497-5p at the acute phase was positively correlated with RBD-lgG antibody response $(r=0.48, P=0.038)$.

Conclusions: Our present results suggested that the expression level of ISR-miRNAs was not only associated with acute SARS-CoV-2 infection but also with RBD-lgG antibody response at the recovery phase of COVID-19. Future studies should be performed to explore the biological significance of ISR-miRNAs in SARS-CoV-2 infection.
\end{abstract}

Keywords: SARS-CoV-2, COVID-19, MicroRNA, RBD-IgG, IFN-I signaling pathway

*Correspondence: njym08@163.com; cdong@suda.edu.cn †Jing Wu, Xingxiang Liu and Jianguo Shao have contributed equally to this work

${ }^{1}$ Department of Epidemiology and Statistics, School of Public Health, Medical College of Soochow University, Soochow, China

${ }^{5}$ Department of Infectious Diseases, The First Affiliated Hospital of Nanjing Medical University, Nanjing, China

Full list of author information is available at the end of the article

\section{Background}

Coronavirus disease 2019 (COVID-19), caused by severe acute respiratory syndrome coronavirus-2 (SARS$\mathrm{CoV}-2$ ), has brought a huge challenge to more than 200 countries and regions [1]. According to the COVID-19 Data Repository by The Center for Systems Science and Engineering at Johns Hopkins University, as of 6 August 
2021, there have been more than 200 million laboratoryconfirmed cases of COVID-19 with 4,289,796 deaths [2].

Type I interferon (IFN-I) exists in vertebrates and triggers the Januskinase/signal transducer and activator of transcription (JAK/STAT) signaling pathway with subsequent induction of IFN-stimulated genes (ISGs) [3]. Previously, mounting studies have shown that IFN-I could affect the life cycle of the virus by regulating the expression of related microRNAs (miRNAs). For example, Aboulnasr et al. reported that IFN- $\alpha / \beta$ could induce the expression of miR-122 in hepatocytes. However, the reduction of miR-122 expression level could weaken the effect of IFN-a/ $\beta$ in inhibiting hepatitis $\mathrm{C}$ virus (HCV) replication [4]. On the other hand, many viruses develop strategies to alter miRNA expression, thereby inhibiting the activity of IFN-I signaling pathway. For example, the results from the sequence alignment indicated that the presence of putative miRNA target sites for the IFN-Iinduced miRNAs located in strictly conserved areas of the HCV genome. Pedersen et al. further confirmed that the expression levels of several miRNAs including miR196, miR-296, miR-351, miR-431 and miR-448 might be affected by binding to the complementary sequences in HCV genome [5].

During the past year, several thousand studies have investigated the epidemiologic, clinical, biological and radiological characteristics of SARS-CoV-2 infection [68]. However, the effects of IFN-I signaling-related miRNAs (ISR-miRNAs) on the virus infection have not yet been fully understood. Based on the prediction results from miRDB and miRPathDB, twelve candidate binding sites for ISR-miRNAs in the genome of SARS-CoV-2 have been identified $[9,10]$. In this study, the expression characteristics of these ISR-miRNAs during acute SARS$\mathrm{CoV}-2$ infection and their associations with receptorbinding domain (RBD) IgG antibody response at the recovery phase were further analyzed.

\section{Materials and methods}

\section{Study participants and data collection}

Between January 2020 and May 2020, 29 COVID-19 patients at the acute phase of COVID-19 and 29 gender and age ( \pm 5 years) matched healthy controls were recruited from Huai'an Fourth hospital. All COVID-19 patients (26 mild and three severe cases) were positive for SARS-CoV-2 RNA in pharyngeal swab specimens and diagnosed according to the "New Coronavirus Pneumonia Prevention and Control Program ( $5^{\text {th }}$ version)" published by the National Health Commission of China [11]. In addition, the healthy controls were laboratory-confirmed cases on the basis of negative qRT-PCR results for SARS-CoV-2 in swab samples. In this study, patients who co-infected with other viruses were excluded. This study was approved by the ethics committee of Huaian Fourth Hospital, Huai'an, China (HASY2020004), and conducted in accordance with the Declaration of Helsinki. All participants signed informed consent forms.

The demographic characteristics of COVID-19 patients and controls, including age, gender and exposure history were collected by face-to-face interview. In addition, the data about clinical signs, symptoms, potential comorbidities and laboratory indices during the acute phase of infection were extracted retrospectively from the electronic medical record system. The incubation period of the disease was defined as the time from exposure to the onset of illness, which was estimated among the patients who could provide the exact date of close contact with individuals confirmed or suspected SARS$\mathrm{CoV}-2$ infection.

\section{Blood sample collection}

Five milliliters of blood samples were collected from each COVID-19 patient within $24 \mathrm{~h}$ of hospitalization (acute phase) and three months after discharge (recovery phase), respectively. In addition, five milliliters of fasting blood samples were also collected from the recruited ageand gender-matched healthy controls. All blood samples were centrifuged at $3000 \mathrm{~g}$ for $10 \mathrm{~min}$. Plasmas were separated and inactivated in a water bath at $56^{\circ} \mathrm{C}$ for $30 \mathrm{~min}$, and then stored at $-80^{\circ} \mathrm{C}$ as quickly as possible.

\section{ISR-miRNA selection}

The complete genome of SARS-CoV-2 strain (NC_045512.2) retrieved from the GenBank database was used as a reference sequence. The miRDB (http://www. mirdb.org/) was firstly used to identify miRNAs which can target the virus genome. The miRNAs with more than 95 of the target score were primarily included $[12,13]$. Then, miRPathDB (https://mpd.bioinf.uni-sb.de/overview.html) was used to identify miRNAs related to the JAK-STAT pathway [14]. After conducted a systematic literature review to identify the ISR-miRNAs using the following terms "JAK", "STAT", and "JAK/STAT" in PubMed, twelve miRNAs were finally selected for the present analysis: let7c-5p, miR-15a-5p, miR-15b-5p, miR-29b-3p, miR-30b-5p, miR-146b-3p, miR-148a-3p, miR-186-5p, miR-409-3p, miR-497-5p, miR-548c-5p and miR-1246. The detailed information about the selected ISR-miRNAs was listed in Additional file 1: Table S1.

\section{Plasma RNA extraction}

Total RNA was isolated from each plasma sample with a commercial RNA extraction and purification kit (MACHEREYNAGEL SA, France) according to the manufacturer's protocol. To warrant consistency in the experimental procedures, exogenous cel-miR-39 
(miDETECT ${ }^{\mathrm{TM}}$ miRNA External Control, RiboBio, China) was spiked into each sample before RNA extraction and used as an internal control for normalizing miRNA expression levels. The concentration of RNA was measured at OD260/280 by a NanoDrop ND-1000 spectrophotometer (Thermo Scientific, Wilmington, Delaware) and kept at $-20^{\circ} \mathrm{C}$ until use.

\section{ISR-miRNA quantitation analysis}

ISR-miRNA quantitation analysis was performed on a Q6 Real-Time System (Applied Biosystems) using the SYBR Green-based real-time detection method. The miDETECTA Track ${ }^{\mathrm{TM}}$ miRNA qRT-PCR Starter Kit, the upstream and downstream primers of selected ISR-miRNAs were ordered from RiboBio Corporation (Guangzhou, China) [15]. The product numbers of the primers for ISR-miRNAs quantitation were showed in Additional file 2: Table S2. The reaction system for ISR-miRNA Poly(A) tailing contained $1 \mu \mathrm{g}$ of total small RNA, $2 \mu \mathrm{L}$ $5 \times \operatorname{Poly}(\mathrm{A})$ polymerase buffer, $1 \mu \mathrm{L}$ Poly(A) polymerase and RNase-free water up to $10 \mu \mathrm{L}$. The reaction system for reverse transcription contained $4 \mu \mathrm{L}$ RTase mix, $4 \mu \mathrm{L} 5 \times$ RTase buffer, $2 \mu \mathrm{L}$ Uni-RT Primer and 10 $\mu \mathrm{L}$ Poly(A) Tailing product. $20 \mu \mathrm{L}$ reaction system for real-time quantitative PCR containing $0.5 \mu \mathrm{L}$ Forward Primer $(10 \mu \mathrm{M}), 0.5 \mu \mathrm{L}$ Uni-Reverse Primer $(10 \mu \mathrm{M}), 10$ $\mu \mathrm{L} 2 \times$ SYBR Green Mix, $0.04 \mu \mathrm{L}$ ROX Reference Dye, 2 $\mu \mathrm{L}$ cDNA and RNase-free water. The cycle threshold (CT value) was defined as the number of cycles required for the fluorescent signal to cross the threshold. As described above, cel-miR-39 was selected as an endogenous control for miRNA expression analysis. The expression of ISR-miRNA relative to cel-miR-39 miRNA was reported as $\mathrm{dCT}(\triangle \mathrm{CT})$, which was calculated by subtracting the $\mathrm{Ct}$ of cel-miR-39 from the Ct of target ISR-miRNA. The relative quantitative of each ISR-miRNA between the two groups was calculated using $2^{-(\Delta \mathrm{CT})}$.

\section{Competitive ELISA for RBD-IgG antibody detection}

The competitive ELISA steps were carried out using "RBD-IgG antibody detection kit" (Beijing, China) according to the manufacturer's introduction. Briefly, 96-well Corning Costar high binding plates were coated with SARS-CoV-2 spike RBD protein at a concentration of $0.1 \mu \mathrm{g}$ per well. For the RBD-IgG antibody measurement, $50 \mu \mathrm{L}$ of 1:50 diluted plasma sample and $50 \mu \mathrm{L}$ of HRP-conjugated ACE2-mFc $(0.2 \mu \mathrm{g} / \mathrm{mL})$ were added into each well. Meanwhile, two negative, two positive plasma controls and two blank wells were included on each plate, respectively. After incubated for $30 \mathrm{~min}$, the plate was washed three times and TMB substrate solution was added. The reaction was stopped after $15 \mathrm{~min}$ by the addition of $2 \mathrm{M} \mathrm{H}_{2} \mathrm{SO}_{4}$. The $\mathrm{OD}$ (optical density) at $450 \mathrm{~nm}$ was measured with an EMax Plus microplate reader (Molecular Devices, San Jose, CA). Results were expressed as percent inhibition (PI), calculated using the following formula: PI $=100 \% \times[1-($ sample OD-blank OD)/(negative control OD-blank OD)]. The sample with more than $25 \%$ of PI value was considered as anti-RBD IgG positive.

\section{Statistical analysis}

All statistical analyses were performed using SAS software (version 9.4). Two-sided $P<0.05$ was considered statistically significant. Differences in the relative expression levels of ISR-miRNAs between COVID-19 patients and healthy controls were compared via Mann-Whitney $U$ test. Spearman correlation was done to measure the correlations between the plasma ISR-miRNA level and clinically relevant parameters at the acute phase of COVID-19, as well as the RBD-IgG antibody levels at the recovery phase.

\section{Results}

Demographic and clinical characteristics of participants

As the results shown in Table 1, the mean age of COVID19 patients and healthy controls was $47.45 \pm 15.72$ and $48.34 \pm 13.50$ years, respectively. Compared with healthy controls, patients with COVID-19 presented lower lymphocyte counts $(Z=3.86, P<0.001)$ and platelet counts $(\mathrm{Z}=2.80, P=0.005)$. In addition, three, nine and one COVID-19 patients had preexisting diabetes, hypertension and renal insufficiency, respectively. The most common symptom at the onset of illness was fever (75.86\%). The median incubation period of the disease was 5 days and the virus nucleic acid test turned negative about 7 days after admission.

\section{Expression profiles of ISR-miRNAs at the acute phase of COVID-19}

The relative expression levels of twelve ISR-miRNAs in the controls and COVID-19 patients during the acute phase were listed in Table 2. Compared with the healthy controls, COVID-19 patients exhibited higher expression levels of miR-29b-3p $(Z=3.15, P=0.002)$ and miR-1246 $(\mathrm{Z}=4.98, P<0.001)$. However, the mean expression levels of miR-186-5p and miR-15a-5p in COVID-19 patients were significantly lower than those in controls. No significant differences in remaining ISR-miRNAs expression were observed between healthy controls and COVID-19 patients at the acute phase.

\section{Association of ISR-miRNAs with clinical parameters at the acute phase of COVID-19}

The correlations between relative levels of ISR-miRNAs and clinically relevant parameters were exhibited 
Table 1 Demographic and clinical characteristics of included participants

\begin{tabular}{|c|c|c|c|c|}
\hline Characteristics & Healthy controls $(n=29)$ & COVID-19 $(n=29)$ & $t / Z / X^{2}$ & $P$ \\
\hline Age, years & $48.34 \pm 13.50$ & $47.45 \pm 15.72$ & 0.23 & 0.817 \\
\hline Male, n (\%) & $14(48.28)$ & $17(58.62)$ & 0.62 & 0.430 \\
\hline $\mathrm{BMI}, \mathrm{kg} / \mathrm{cm}^{2}$ & $24.41 \pm 4.33$ & $26.40 \pm 3.84$ & 1.85 & 0.070 \\
\hline \multicolumn{5}{|l|}{ Signs and symptoms } \\
\hline Fever & NA & $22(75.86)$ & & \\
\hline Cough & NA & $14(48.27)$ & & \\
\hline Myalgia or fatigue & NA & $2(6.90)$ & & \\
\hline Expectoration & NA & $2(6.90)$ & & \\
\hline Headache & NA & $1(3.44)$ & & \\
\hline Oppression in chest & NA & $2(6.90)$ & & \\
\hline Incubation period (days) & NA & $5.0(4.0,7.0)$ & & \\
\hline Days from first admission to transfer & NA & $7.0(4.0,8.5)$ & & \\
\hline \multicolumn{5}{|l|}{ Underlying } \\
\hline Hypertension & $0(0.00)$ & $9(31.03)$ & & \\
\hline Diabetes & $0(0.00)$ & $3(10.34)$ & & \\
\hline \multicolumn{5}{|l|}{ Laboratory findings } \\
\hline White blood cell, $\times 10^{9} / \mathrm{L}$ & $5.77(5.13,6.17)$ & $5.27(4.21,6.80)$ & 0.96 & 0.335 \\
\hline Neutrophil, $\times 10^{9} / \mathrm{L}$ & $3.28(2.46,4.02)$ & $3.32(2.49,4.93)$ & 0.50 & 0.619 \\
\hline Lymphocyte, $\times 10^{9} / \mathrm{L}$ & $1.98(1.61,2.12)$ & $1.07(0.81,1.49)$ & 3.86 & $<0.001$ \\
\hline Hemoglobin, g/L & $132(122,149)$ & $144(133,151)$ & 1.79 & 0.074 \\
\hline Platelet, $\times 10^{9} / \mathrm{L}$ & $214(182,253)$ & $176(149,198)$ & 2.80 & 0.005 \\
\hline Fasting plasma glucose, mmol/L & NA & $6.64 \pm 2.23$ & & \\
\hline CD4 + T cell $(\mu \mathrm{L})$ & NA & $433(272,743)$ & & \\
\hline CD8 + T cell $(\mu \mathrm{L})$ & NA & $292(171,455)$ & & \\
\hline
\end{tabular}

Table 2 Expression profiles of ISR-miRNAs in healthy controls and acute COVID-19 patients

\begin{tabular}{|c|c|c|c|c|}
\hline ISR-miRNAs & Healthy controls $(n=29)$ & COVID-19 $(n=29)$ & z & $P$ \\
\hline hsa-let-7c-5p & $0.22(0.05,0.60)$ & $0.21(0.05,0.44)$ & 0.00 & 1.000 \\
\hline hsa-miR-29b-3p & $0.06(0.01,0.25)$ & $0.33(0.11,0.78)$ & 3.15 & 0.002 \\
\hline hsa-miR-30b-5p & $0.28(0.10,0.79)$ & $0.58(0.22,1.60)$ & 1.59 & 0.113 \\
\hline hsa-miR-186-5p & $0.35(0.17,0.94)$ & $0.06(0.03,0.19)$ & 3.52 & $<0.001$ \\
\hline hsa-miR-15a-5p & $0.13(0.04,0.53)$ & $0.02(0.01,0.11)$ & 2.26 & 0.024 \\
\hline hsa-miR-15b-5p & $0.05(0.02,0.26)$ & $0.06(0.02,0.13)$ & 0.00 & 1.000 \\
\hline hsa-miR-148a-3p & $0.12(0.02,0.29)$ & $0.06(0.03,0.15)$ & 0.99 & 0.322 \\
\hline hsa-miR-146b-3p & $0.11(0.03,0.35)$ & $0.15(0.07,0.27)$ & 1.01 & 0.312 \\
\hline hsa-miR-409-3p & $0.11(0.03,0.34)$ & $0.24(0.06,0.34)$ & 1.23 & 0.218 \\
\hline hsa-miR-497-5p & $0.03(0.01,0.11)$ & $0.05(0.03,0.12)$ & 0.75 & 0.456 \\
\hline hsa-miR-548c-5p & $0.06(0.02,0.28)$ & $0.09(0.04,0.28)$ & 1.44 & 0.151 \\
\hline hsa-miR-1246 & $0.06(0.04,0.11)$ & $0.53(0.19,1.12)$ & 4.98 & $<0.001$ \\
\hline
\end{tabular}

in Fig. 1. The results showed that the plasma level of miR-30b-5p was negatively correlated with CD $4+\mathrm{T}$ cell counts in patients with acute SRAS-CoV-2 infection $(\mathrm{r}=-0.41, P=0.027)$. In addition, the relative concentration of miR-30b-5p was marginally correlated with fasting plasma glucose level $(r=0.37, P=0.052)$. However, no significant correlation was observed between other ISR-miRNAs expression and clinically relevant parameters of COVID-19 patients at the acute phase. 


\begin{tabular}{|c|c|c|c|c|c|c|c|c|c|c|c|c|}
\hline-0.06 & 0.14 & & 0.14 & -0.35 & 0.04 & -0.01 & & 0.02 & 0.11 & -0.19 & & White blood cell \\
\hline 0.04 & 0.10 & -0.12 & 0.16 & & 0.08 & -0.03 & 0.21 & -0.05 & 0.18 & -0.18 & & Neutrophil \\
\hline-0.18 & 0.17 & & -0.00 & -0.18 & -0.05 & 0.06 & -0.03 & 0.15 & -0.12 & -0.01 & -0.14 & Lymphocyte \\
\hline 0.04 & -0.01 & 0.01 & 0.05 & -0.11 & 0.21 & -0.10 & 0.02 & -0.17 & 0.27 & 0.10 & -0.01 & Hemoglobin \\
\hline 0.00 & 0.07 & -0.14 & -0.18 & -0.04 & -0.06 & 0.22 & -0.03 & 0.26 & -0.10 & & & Platelet \\
\hline 0.06 & 0.04 & 0.37 & 0.19 & 0.21 & 0.09 & 0.26 & 0.18 & -0.06 & 0.04 & 0.17 & -0.16 & Fasting plasma glucose \\
\hline & -0.17 & $-0,41$ & -0.08 & -0.13 & -0.08 & -0.11 & $x$ & 0.09 & -0.09 & & -0.12 & CD4+ T cell \\
\hline-0.16 & -0.01 & -0.32 & 0.07 & & -0.03 & -0.02 & 0.00 & 0.21 & -0.07 & -0.07 & -0.22 & CD8+ T cell \\
\hline $\begin{array}{l}\overline{\mathbb{9}} \\
\dot{0} \\
0 \\
\dot{0} \\
\dot{0}\end{array}$ & 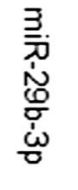 & 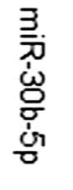 & 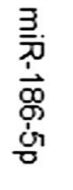 & 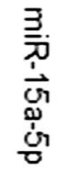 & 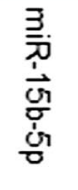 & 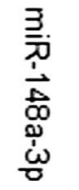 & 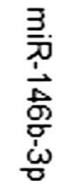 & $\begin{array}{l}3 \\
00 \\
0 \\
0 \\
0 \\
0 \\
0 \\
0\end{array}$ & 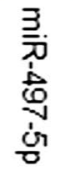 & 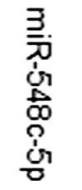 & $\begin{array}{l}\stackrel{3}{D} \\
\stackrel{\vec{N}}{N} \\
\text { 离 }\end{array}$ & \\
\hline
\end{tabular}

Fig. 1 Spearman correlation between the relative expression levels of twelve ISR-miRNAs and clinical parameters. The values in each square are correlation coefficient (r) of each group samples. The dendrogram on the right reveals the sample's correlation

Table 3 Correlation between clinical parameters and RBD-lgG antibody response

\begin{tabular}{lrl}
\hline & $\mathbf{r}$ & $\boldsymbol{P}$ \\
\hline White blood cell & -0.15 & 0.461 \\
Neutrophil & 0.12 & 0.541 \\
Lymphocyte & -0.62 & 0.0004 \\
Hemoglobin & -0.10 & 0.615 \\
Platelet & -0.18 & 0.368 \\
Fasting plasma glucose & 0.26 & 0.174 \\
CD4+T cell & -0.29 & 0.138 \\
CD8+T cell & -0.43 & 0.023 \\
\hline
\end{tabular}

\section{Association between ISR-miRNAs expression and RBD-IgG antibody response}

Among 28 patients who provided enough convalescent plasma samples, RBD-IgG antibodies were detected in 27 COVID-19 patients using competitive ELISA. The highest and lowest PI values were $93.0 \%$ and $41.2 \%$ (median PI: 77.5\%), respectively. As the results shown in Table 3, the counts of lymphocytes and CD $8+T$ cells at the acute phase of the disease was negatively correlated with the level of RBD-IgG antibody at the recovery phase, with $\mathrm{r}$ values of $-0.62(P=0.0004)$ and $-0.43(P=0.023)$, respectively.

The association between the relative expression levels of plasma ISR-miRNAs at the acute phase of disease and the level of RBD-IgG antibody at the recovery phase was shown in Table 4. The plasma level of miR-497-5p at the acute phase of COVID-19 was positively correlated to RBD-IgG antibody response at the recovery phase
Table 4 Correlation between the expression levels of ISRmiRNAs and RBD-lgG antibody response

\begin{tabular}{lcl}
\hline ISR-miRNAs & \multicolumn{1}{l}{} & $\boldsymbol{P}$ \\
\hline hsa-let-7c-5p & 0.24 & 0.229 \\
hsa-miR-29b-3p & -0.08 & 0.689 \\
hsa-miR-30b-5p & 0.04 & 0.836 \\
hsa-miR-186-5p & 0.10 & 0.635 \\
hsa-miR-15a-5p & 0.28 & 0.257 \\
hsa-miR-15b-5p & -0.16 & 0.501 \\
hsa-miR-148a-3p & -0.06 & 0.778 \\
hsa-miR-146b-3p & -0.004 & 0.986 \\
hsa-miR-409-3p & -0.03 & 0.896 \\
hsa-miR-497-5p & 0.48 & 0.038 \\
hsa-miR-548c-5p & 0.09 & 0.676 \\
hsa-miR-1246 & 0.04 & 0.850 \\
\hline
\end{tabular}

of disease $(r=0.48, P=0.038)$. However, no significant correlation was observed between other ISR-miRNA concentrations and RBD-IgG antibody response in the COVID-19 patients.

\section{Discussion}

Previously, several studies have indicated that ISR-miRNAs were significantly associated with SARS-CoV-2 infection [3-5]. For example, Centa A et al. reported that deregulated expression of miR-26a-5p, miR-29b-3p and miR-34a-5p was associated with endothelial dysfunction in post-mortem lung biopsies of COVID-19 patients [16]. Additionally, in a computational and bioinformatic analysis, Khan AT 
et al. speculated that miR-1246 might be upregulated in the respiratory distress caused by COVID-19 [17]. In the present study, our results further showed that the expression of miR-29b-3p and miR-1246 were significantly upregulated at the acute phase of COVID-19, suggesting that miR29b-3p and miR-1246 might play an important role in the pathogenesis of acute SARS-CoV-2 infection.

Our present study observed that the expression levels of miR-186-5p and miR-15a-5p significantly decreased at the acute phase of COVID-19. Following in silico target prediction and pathway enrichment analyses, Zhao et al. suggested that miR-186-5p was depleted in retroviral infection. Similarly, Wu et al. reported that overexpressed miR-186 could inhibit the JAK/STAT signaling pathway in vitro [18]. In addition, the increased miR-186-5p expression could inhibit HIV infection by immunoregulation and $\mathrm{T}$ cell regulation [19]. The results from the most recent study also reported that miR-15b-5p were significantly downregulated in hamster lung samples infected by SARS-CoV-2 [20]. Considering that the miR15 family members (i.e., miR-15a, 15b) possess the same seed sequence and have the same target genes, the present results further suggested that downregulated expression of miR-186-5p and miR-15a-5p might be helpful for the activation of IFN-I signaling pathway at the acute phase of SARS-CoV-2 infection.

Recently, Zheng et al. reported that the activation and differentiation of $T$ cells are associated with various clinical features in patients with COVID-19 [21]. In a retrospective single-center study, Liu et al. further reported that CD4+ and CD8 + T cells were significantly related to the severity of SARS-CoV-2 infection [22]. Moreover, reduced CD3+, CD4+ and CD8 + T cell counts could reflect the severity of the COVID-19 [23]. In our study, the results showed the expression level of miR-30b-5p was negatively correlated with $\mathrm{CD} 4+\mathrm{T}$ cell counts. These findings were shown from the side that miR-30b-5p might be associated with the severity of COVID-19 patients. Additionally, our present results showed that the level of miR-30b-5p increased with the level of fasting plasma glucose in COVID-19 patients at the acute phase. Considering that miR-30b-5p was much higher in patients with type 2 diabetes mellitus (T2DM) and the severity of COVID-19 was significantly associated with diabetes [24, 25], more studies should be conducted to clarify the effects of miR-30b-5p on SARS$\mathrm{CoV}-2$ infection in patients with and without T2DM.

Currently, a large number of studies have reported that the interaction between RBD located at the spike protein of SARS-CoV-2 and the receptor ACE2 on host cells is essential for viral entry [26, 27]. Moreover, antibodies against RBD at the recovery phase of COVID-19 present neutralizing activity because they can block the interaction between ACE2 and viral spike protein [28]. For example, Premkumar et al. reported that antibodies targeting RBD accounted for more than $90 \%$ of neutralizing activity in convalescent serum [29]. In this study, the results of competitive ELISA showed that 27/28 COVID19 patients developed RBD-IgG antibodies at the recovery phase. Moreover, the plasma level of miR-497-5p at the acute phase positively correlated with RBD-IgG antibody response. However, the counts of lymphocyte and $\mathrm{CD} 8+\mathrm{T}$ cells during the acute phase were inversely correlated with RBD-IgG antibody response, indicating that the mechanisms of miR-497-5p expression involved in RBD-IgG response should be further explored.

Our present study has several limitations. First, considering that only 29 patients were included in this study, caution should be taken when interpreting the present findings. Second, since the disease progression and antibody response might be influenced by various risk factors (age, comorbidity disease such as hypertension and diabetes) [30-32], the effects of the ISR-miRNAs and interactions of other risk factors on SARS-CoV-2 infection should also be carefully verified in future. Last, although our study was the first time to report that miR-29b-3p, miR-1246, miR-186-5p and miR15a-5p expressions were significantly altered during acute SARS-CoV-2 infection, the difference in plasma ISR-miRNAs between patients with mild and severe infection could not be clarified because of the limited sample size.

\section{Conclusions}

The expression level of ISR-miRNAs was not only associated with acute SARS-CoV-2 infection but also with RBD-IgG antibody response at the recovery phase of the disease. However, considering that the limited sample size in this study, further studies including more patients are needed to validate our present findings and to explore the biological significance of ISR-miRNAs during SARS-CoV-2 infection.

\begin{abstract}
Abbreviations
COVID-19: Coronavirus disease 2019; SARS-CoV-2: Severe acute respiratory syndrome coronavirus-2; IFN-I: Type I interferon; JAK-STAT: Januskinase/signal transducer and activator of transcription; ISGs: IFN-stimulated genes; HCV: Hepatitis C virus; microRNA: MiRNA; ISR-miRNAs: IFN-I signaling-related miRNAs; CT: Cycle threshold; OD: Optical density; RBD: Receptor-binding domain; PI: Percent inhibition; T2DM: Type 2 diabetes mellitus.
\end{abstract}

\section{Supplementary Information}

The online version contains supplementary material available at https://doi. org/10.1186/s12985-021-01717-7.

Additional file 1: Table S1. List of human miRNAs with target gene and miRNAs seed binding site on COVID-19 isolates.

Additional file 2: Table S2. Product number of the primers for ISRmiRNAs quantitation. Footnote: RiboBio Corporation (Guangzhou, China), https://www.ribobio.com/. 


\section{Acknowledgements}

We gratefully acknowledge Yun Zhang, Yonghong Zhang and other volunteers who proofed reading the article.

\section{Authors' contributions}

CD: Conceptualization, Methodology, review and editing; MY: Methodology, review and editing; JW: Writing — original draft, investigation, Formal analysis; XL: Writing —original draft, investigation, Formal analysis; JS: Writing—original draft, investigation, Formal analysis; YZ: Investigation, Data curation; RL: Investigation, Data curation; YX: Data curation, Resources; LW: Data curation, Resources; HX: Data curation, Resources; HZ: Investigation, Validation; LY: Investigation, Validation. All authors read and approved the final manuscript.

\section{Funding}

This work was supported by the National Natural Science Foundation of China (No. 81773507), Special Foundation for COVID-19 from Soochow University (JJ13900420), The Foundation of Technology Program in Soochow (SYS2020049), and Special Foundation for COVID-19 from Nantong (HS2020002).

\section{Availability of data and materials}

The data and materials included in the present study could be provided by Chen Dong (cdong@suda.edu.cn) and Jing Wu (20194247006@stu.suda.edu. $\mathrm{cn}$ ) on reasonable request.

\section{Declarations}

\section{Ethics approval and consent to participate}

This study was approved by the ethics committee of Huai'an Fourth Hospital, Huai'an, China (Ethics Certification Number: HASY2020004), and written informed consent was obtained from each participant.

\section{Consent for publication}

Not applicable.

\section{Competing interests}

The authors declare that they have no competing interest.

\section{Author details}

1 Department of Epidemiology and Statistics, School of Public Health, Medical College of Soochow University, Soochow, China. ${ }^{2}$ Department of Clinical Laboratory, Huai'an Fourth People's Hospital, Huai'an, China. ${ }^{3}$ Nantong Third People's Hospital, Nantong, China. ${ }^{4}$ Suzhou Industrial Park Centers for Disease Control and Prevention, Soochow, China. ${ }^{5}$ Department of Infectious Diseases, The First Affiliated Hospital of Nanjing Medical University, Nanjing, China.

Received: 25 August 2021 Accepted: 27 November 2021

Published online: 07 December 2021

\section{References}

1. O'Driscoll M, Ribeiro Dos Santos G, Wang L, et al. Age-specific mortality and immunity patterns of SARS-CoV-2. Nature. 2021;590(7844):140-5.

2. COVID-19 Data Repository by the Center for Systems Science and Engineering (CSSE) at Johns Hopkins University. https://www.arcgis.com/ apps/opsdashboard/index.htm|\#/bda7594740fd40299423467b48e9ecf6. Accessed 6 Aug 2021.

3. Wang Y, Zhu P, Qiu J, Wang J, Zhu H, Zhu Y, et al. Identification and characterization of interferon signaling-related microRNAs in occult hepatitis B virus infection. Clin Epigenetics. 2017;9:101.

4. Aboulnasr F, Hazari S, Nayak S, Chandra PK, Panigrahi R, Ferraris P, et al. IFN- $\lambda$ inhibits miR-122 transcription through a Stat3-HNF4a inflammatory feedback loop in an IFN-a resistant HCV cell culture system. PLoS ONE. 2015;10(12):e0141655.

5. Pedersen IM, Cheng G, Wieland S, Volinia S, Croce CM, Chisari FV, et al. Interferon modulation of cellular microRNAs as an antiviral mechanism. Nature. 2007:449(7164):919-22.

6. Hu B, Guo H, Zhou P, Shi ZL. Characteristics of SARS-CoV-2 and COVID-19. Nat Rev Microbiol. 2021;19(3):141-54.
7. Guan WJ, Ni ZY, Hu Y, Liang WH, Ou CQ, He JX, et al. Clinical characteristics of coronavirus disease 2019 in China. N Engl J Med. 2020;382(18):1708-20.

8. Jiang H, Guo W, Shi Z, Jiang H, Zhang M, Wei L, et al. Clinical imaging characteristics of inpatients with coronavirus disease-2019 in Heilongjiang Province, China: a retrospective study. Aging (Albany NY). 2020;12(14):13860-8.

9. Arisan ED, Dart A, Grant GH, Arisan S, Cuhadaroglu S, Lange S, et al. The prediction of miRNAs in SARS-CoV-2 genomes: hsa-miR databases identify 7 key miRs linked to host responses and virus pathogenicity-related KEGG pathways significant for comorbidities. Viruses. 2020;12(6):614.

10. Fulzele S, Sahay B, Yusufu I, Lee TJ, Sharma A, Kolhe R, et al. COVID-19 virulence in aged patients might be impacted by the host cellular microRNAs abundance/profile. Aging Dis. 2020;11(3):509-22.

11. Diagnosis and treatment of new coronavirus pneumonitis. (trial version 5): National Health Commission of the People's Republic of China. http:// www.nhc.gov.cn/yzygj/s7653p/202002/3b09b894ac9b4204a79db5b89 12d4440.shtml. Accessed 8 Aug 2021.

12. Chen $Y$, Wang $X$. miRDB: an online database for prediction of functional microRNA targets. Nucleic Acids Res. 2020:48(D1):D127-31.

13. Wang JD, Zhou HS, Tu XX, He Y, Liu QF, Liu Q, et al. Prediction of competing endogenous RNA coexpression network as prognostic markers in AML. Aging (Albany NY). 2019;11(10):3333-47.

14. Kehl T, Kern F, Backes C, Fehlmann T, Stöckel D, Meese E, et al. miRPathDB 2.0: a novel release of the miRNA pathway dictionary database. Nucleic Acids Res. 2020;48(D1):D142-7.

15. Liu L, Liu Y, Feng C, Chang J, Fu R, Wu T, et al. Lithium-containing biomaterials stimulate bone marrow stromal cell-derived exosomal miR-130a secretion to promote angiogenesis. Biomaterials. 2019;192:523-36.

16. Centa A, Fonseca AS, Ferreira SGDS, Azevedo MLV, Vaz de Paula CB, Nagashima S, et al. Deregulated miRNA expression is associated with endothelial dysfunction in post-mortem lung biopsies of COVID-19 patients. Am J Physiol Lung Cell Mol Physiol. 2020;320(3):L405-12.

17. Khan AT, Khalid Z, Zahid H, Yousaf MA, Shakoori AR. A computational and bioinformatic analysis of ACE2: an elucidation of its dual role in COVID-19 pathology and finding its associated partners as potential therapeutic targets. J Biomol Struct Dyn. 2020;1-17. https://doi.org/10.1080/07391 102.2020.1833760

18. Wu DM, Wen X, Wang YJ, Han XR, Wang S, Shen M, et al. Effect of microRNA-186 on oxidative stress injury of neuron by targeting interleukin 2 through the Janus kinase-signal transducer and activator of transcription pathway in a rat model of Alzheimer's disease. J Cell Physiol. 2018;233(12):9488-502.

19. Zhao Z, Muth DC, Mulka K, Liao Z, Powell BH, Hancock GV, et al. miRNA profiling of primate cervicovaginal lavage and extracellular vesicles reveals miR-186-5p as a potential antiretroviral factor in macrophages. FEBS Open Bio. 2020;10(10):2021-39.

20. Kim WR, Park EG, Kang KW, Lee SM, Kim B, Kim HS. Expression analyses of microRNAs in hamster lung tissues infected by SARS-CoV-2. Mol Cells. 2020;43(11):953-63.

21. Zheng HY, Xu M, Yang CX, Tian RR, Zhang M, Li JJ, et al. Longitudinal transcriptome analyses show robust $T$ cell immunity during recovery from COVID-19. Signal Transduct Target Ther. 2020;5(1):294.

22. Liu Z, Long W, Tu M, Chen S, Huang Y, Wang S, et al. Lymphocyte subset (CD4+, CD8+) counts reflect the severity of infection and predict the clinical outcomes in patients with COVID-19. J Infect. 2020;81 (2):318-56.

23. Liu R, Wang Y, Li J, Han H, Xia Z, Liu F, et al. Decreased T cell populations contribute to the increased severity of COVID-19. Clin Chim Acta. 2020;508:110-4.

24. Stępień EŁ, Durak-Kozica M, Kamińska A, Targosz-Korecka M, Libera M, Tylko G, et al. Circulating ectosomes: determination of angiogenic microRNAs in type 2 diabetes. Theranostics. 2018:8(14):3874-90.

25. Yang $S$, Chen $X$, Yang M, Zhao X, Chen Y, Zhao H, et al. The variant at TGFBRAP1 is significantly associated with type 2 diabetes mellitus and affects diabetes-related miRNA expression. J Cell Mol Med. 2019;23(1):83-92.

26. Kathiravan MK, Radhakrishnan S, Namasivayam V, Palaniappan S. An overview of spike surface glycoprotein in severe acute respiratory syndromecoronavirus. Front Mol Biosci. 2021;8:637550.

27. Kadam SB, Sukhramani GS, Bishnoi P, Pable AA, Barvkar VT. SARS-CoV-2, the pandemic coronavirus: molecular and structural insights. J Basic Microbiol. 2021;61(3):180-202 
28. Collins DP, Steer CJ. Binding of the SARS-CoV-2 spike protein to the asialoglycoprotein receptor on human primary hepatocytes and immortalized hepatocyte-like cells by confocal analysis. Hepat Med. 2021;13:37-44.

29. Premkumar L, Segovia-Chumbez B, Jadi R, Martinez DR, Raut R, Markmann $A$, et al. The receptor binding domain of the viral spike protein is an immunodominant and highly specific target of antibodies in SARS-CoV-2 patients. Sci Immunol. 2020;5(48):eabc8413.

30. Wu J, Zhang J, Sun X, Wang L, Xu Y, Zhang Y, et al. Influence of diabetes mellitus on the severity and fatality of SARS-CoV-2 (COVID-19) infection. Diabetes Obes Metab. 2020;22(10):1907-14.

31. Zhang J, Wu J, Sun X, Xue H, Shao J, Cai W, et al. Association of hypertension with the severity and fatality of SARS-CoV-2 infection: a meta-analysis. Epidemiol Infect. 2020;148:e106.

32. Savoia C, Volpe M, Kreutz R. Hypertension, a moving target in COVID-19: current views and perspectives. Circ Res. 2021;128(7):1062-79.

\section{Publisher's Note}

Springer Nature remains neutral with regard to jurisdictional claims in published maps and institutional affiliations.

- fast, convenient online submission

- thorough peer review by experienced researchers in your field

- rapid publication on acceptance

- support for research data, including large and complex data types

- gold Open Access which fosters wider collaboration and increased citations

- maximum visibility for your research: over 100M website views per year

At BMC, research is always in progress.

Learn more biomedcentral.com/submissions 